\title{
Cellular Pleomorphic Adenoma
}

National Cancer Institute

\section{Source}

National Cancer Institute. Cellular Pleomorphic Adenoma. NCI Thesaurus. Code C83174.

A pleomorphic adenoma with increased cellularity. 